\title{
Brief
}

\section{Single-stage bilateral minimally invasive approach for pulmonary hydatid disease: An alternative technique}

\author{
Ghulam Nabi Lone, MS, MCh, ${ }^{a}$ Mohd Akbar Bhat, MS, MCh, ${ }^{a}$ Noor Ali, MS, MCh, ${ }^{a}$ Adil Bashir, MS, and

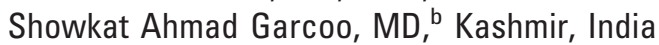

From the Department of Cardiovascular and Thoracic Surgery a and the Department of Anaesthesiology, ${ }^{\mathrm{b}}$ Sher-I-Kashmir Institute of Medical Sciences, Srinagar, Kashmir, India.

Received for publication March 1, 2001; accepted for publication Dec 4, 2001.

Address for reprints: Ghulam Nabi Lone, MS, MCh, PO Box 1222, GPO Srinagar190001, Kashmir, India (E-mail: root@skims ren.in.nic).

J Thorac Cardiovasc Surg 2002;124:1021-4 Copyright $\odot 2002$ by The American Association for Thoracic Surgery

$0022-5223 / 2002 \$ 35.00+0 \quad \mathbf{1 2 / 5 4 / 1 2 2 3 1 5}$

doi: $10.1067 / \mathrm{mtc} .2002 .122315$

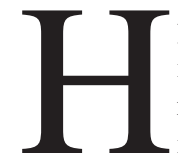

ydatid cystic disease, a parasitic infection caused by Echinococcus granulosus, is characterized by cystic space-occupying lesions in the liver, the lungs, and rarely in other parts of the body. ${ }^{1-3}$ The liver acts as the first filter for larval infection, and the lungs act as the second filter.

The diagnostic hallmark is a well-defined, round, radiopaque shadow with homogeneous density, with little or no parenchymal reaction around the lesion. ${ }^{1}$ An untreated lung hydatid cyst can rupture into the bronchial tree or pleural cavity and may cause anaphylaxis or asphyxiation. In addition, if the cyst becomes infected, lung abscess, empyema, bronchopleural fistula, bronchiectasis, and atelectasis may develop. ${ }^{2}$ Conventionally, unilateral lung hydatid cyst is treated by surgical excision through a posterolateral thoracotomy (standard) with wide exposure, and bilateral lung hydatid cysts require a two-stage approach. ${ }^{4}$ Median sternotomy provides access to both lungs without direct injury to the respiratory muscles. ${ }^{4,5}$

We have created a bilateral anterior muscle-sparing minithoracotomy approach that enables us to shorten hospital stay by offering early and relatively painless postoperative recovery. This technique is economical, cosmetically appealing, and convenient to the patient and family. It also avoids social embarrassment, in contrast to the conventional approach.

\section{Patients and Methods}

A total of 142 patients with pulmonary hydatid cysts were admitted to the Department of Cardiovascular and Thoracic Surgery, Sher-I-Kashmir Institute of Medical Sciences, Srinagar, Kashmir, India, from January 1997 to August 2000. Among 142 patients, 16 had bilateral lung hydatid cysts. Among these 16 cases, 12 patients were selected prospectively and operated on through the minimally invasive approach. Baseline investigations were performed. Blood and sputum cultures were routinely performed to rule out infected cysts. Lung function tests were done before and after the operation. Diagnosis was established by chest radiography (Figure 1), the Casoni intradermal reaction, and serologic studies (enzyme-linked immunosorbent assay). Thoracic computed tomographic scan and bronchoscopy were performed in doubtful cases. Abdominal ultrasonography was routinely done to exclude concurrent intra-abdominal hydatid cyst.

Eight patients had eosinophilia of $4 \%$. The Casoni reaction was positive in 9 cases. Results of enzyme-linked immunosorbent assay were positive in 10 cases. Results of sputum examination (by the Ziehl Neelson technique) were negative for acid-fast bacilli in all cases. Chest radiography was consistent with hydatid cyst in 10 cases, and 2 had radiographically doubtful lesions that required computed tomography and bronchoscopy for confirmation. Abdominal ultrasonography ruled out abdominal hydatidosis in all 12 patients. Only those who did not have hepatic or splenic hydatid cysts were taken for study. The hydatid cyst was located in both lower lobes in 4 patients, in the right upper lobe and left lower lobe in 3 patients, in the oblique fissure on the right side and the left lower lobe in 2 patients, in the bilateral subpulmonic area in 1 patient, in the middle lobe on the right side and the upper lobe on the left side in 1 patient, and in the horizontal fissure and the left lower lobe in 1 patient. The hydatid cysts in all these patients were unilocular in nature. Strict criteria regarding selection of cases for minithoracotomy were followed. 


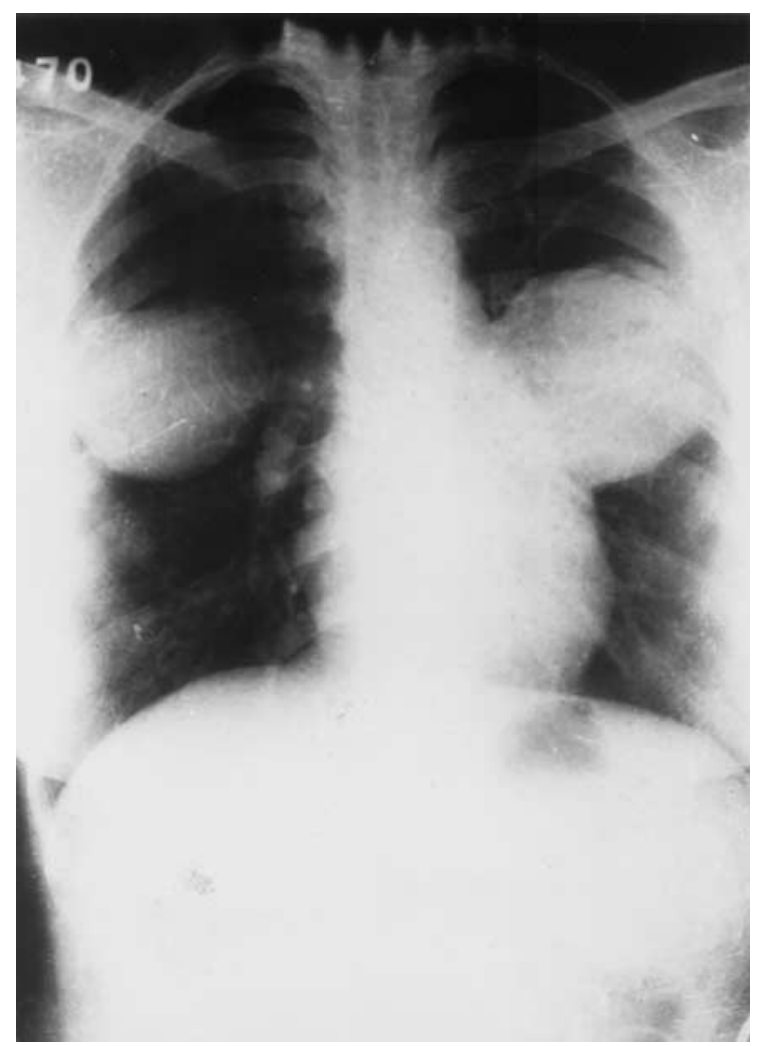

Figure 1. Chest radiograph (posteroanterior view) revealing large, well-defined radiopaque shadows in midzones of both lungs; these proved to be hydatid cysts.

Selection criteria. Selection criteria were as follows:

1. Small to moderately sized cysts $(<10 \mathrm{~cm})$

2. Anteriorly situated cysts

3. Sterile cysts

4. Isolated pulmonary (bilateral) cysts

5. High-risk patients with bilateral disease in the presence of the preceding criteria.

Patients with giant cysts, multilobar cysts, infected cysts, and posteriorly located cysts were approached through posterolateral route and therefore excluded from the study. Patients with associated hepatic or splenic cysts were approached through the transthoracic-transdiaphragmatic route.

\section{Technique}

In the minithoracotomy technique, the patient is draped in the supine posture with both arms extended and supported over the head, exposing the chest widely from suprasternal notch up to the umblicus vertically and from the right to the left midaxillary line horizontally. Single-lung ventilation is ensured in all cases after induction of anesthesia. As a prophylaxis, $200 \mathrm{mg}$ hydrocortisone is injected by the intravenous route in each case. A small incision (4-5 cm long) is made in the fifth intercostal space, lateral to midclavicular line. In young girls a small submammary incision is preferred. The chest is opened through the intercostal space after splitting of the corresponding digitation of the serratus anterior

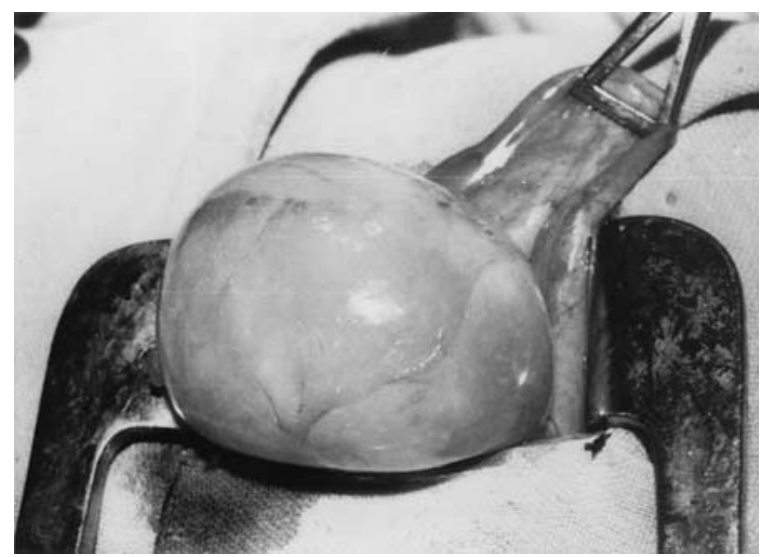

Figure 2. Hydatid cyst being delivered by Barrett technique through small left anterior thoracotomy incision (masked by hydatid cyst) with sequential method.

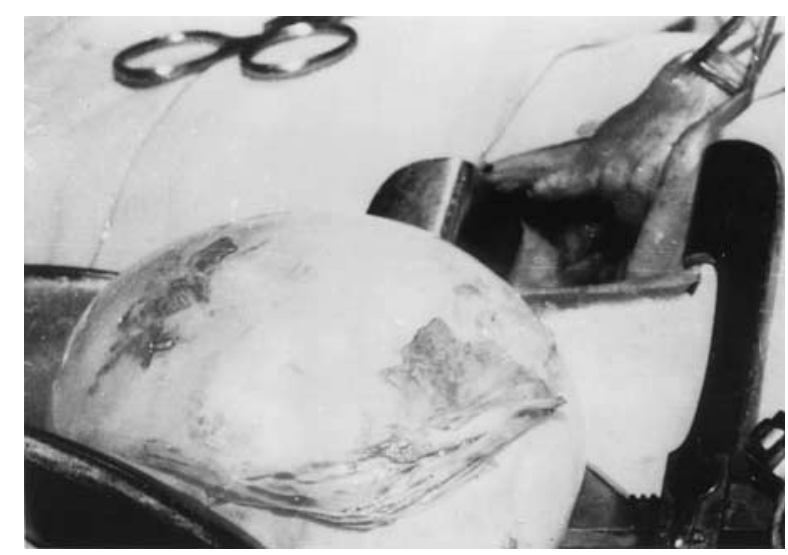

Figure 3. Large hydatid cyst $(8 \times 6 \mathrm{~cm}$ in size $)$ delivered intact. Small thoracotomy incision is now visible. Lung parenchyma is held by Duval forceps, and hydatid cyst has been collected in kidney tray.

simultaneously by two surgeons or sequentially by a single surgeon with a Tuffier rib retractor (blade width $44 \mathrm{~mm}$, blade depth $51 \mathrm{~mm}$ ) or Finochietto rib retractor (blade width $32 \mathrm{~mm}$, blade depth $30 \mathrm{~mm}$ ). The cyst is isolated after division of the adhesions, while the surrounding lung is packed off with gauze packs soaked with a scolicidal solution (hypertonic saline solution). The lung tissue surrounding the cyst is held by Duval forceps and pulled up gently to the level of the skin. The pericyst is incised and separated from the hydatid cyst wall all around without rupturing the cyst. The cyst is delivered by the Barrett technique (Figures 2 and 3). Any bronchial communications are closed with 2-0 silk, and the residual cavity is obliterated by capitonnage (obliteration of the evacuated cyst cavity by circumferential suture technique). We routinely use 3 to 4 rows of 2-0 silk for this purpose, depending on the depth of the cavity. Pericystectomy, if desired, is performed without incising the adventitia by separating the pericyst with the 
cyst in the center from the surrounding normal parenchyma. However, this procedure leads to significant hemorrhage and is timeconsuming.

After obliteration of the dead space and removal of the gauze packs, hemostasis is achieved by electrocautry and a single chest drain is inserted in the thoracic cavity on each side. The chest is closed by the subcuticular suture technique with 3-0 Vicryl suture (Ethicon, Inc, Somerville, NJ) for skin closure. If sequential mode of minithoracotomy is selected, then the previously mentioned procedure is repeated on the contralateral side. We preferred the sequential mode with a $30^{\circ}$ lateral tilt toward the opposite side. After completion of the procedure, the patient is extubated on table and observed for any complications.

Ten patients underwent anterior minithoracotomy on both sides by single surgeon, and 2 were operated on simultaneously on both sides. Seven underwent enucleation and capitonnage, 3 underwent pericystectomy and capitonnage, and 2 underwent segmentectomy on the left side and enucleation and capitonnage on the right side. All patients could be extubated at the end of the procedure.

\section{Results}

On analysis, 16 of 142 patients were found to have bilateral lung hydatidosis $(11.26 \%)$. The male/female ratio was $2: 1$, and the mean age was 20.7 years. The youngest patient was 7 years old, and the oldest was 60 years old. Chest pain was the most common symptom $(75 \%)$, followed by cough $(58.3 \%)$ and hemoptysis $(50 \%)$. Results of chest examination were normal in most cases (58.3\%; Table 1). Mean operative time was 84 minutes (range 65-120 minutes). The mean duration of intercostal tube drainage was 3.6 days (range 2-11 days). The average perioperative blood loss was $360 \mathrm{~mL}$. One patient had a wound infection with Klebsiella pneumoniae, which was treated conservatively. One patient had minimal air leakage for less than 4 days. However, another patient had prolonged air leakage for 11 days. The mortality was zero. The average hospital stay was 4.6 days. Recurrence had not been detected in any patient as of the last follow-up. We did not use albendazole (Zentel) after the operation on a routine basis, unless a strong suspicion of spillage of hydatid fluid arose during the procedure.

We prospectively analyzed data from 142 patients with lung hydatidosis. Sixteen patients had bilateral disease (11.26\%), and 20 had concomitant hepatic and lung cysts (14.8\%). Three of the 16 patients with bilateral disease had multilobar bilateral lung cysts, and 1 patient had extensive bilateral calcified lung hydatidosis and did not need surgical intervention (excluded from study). Our preferred method of cyst removal was enucleation and capitonnage. However, those patients who had cysts located in the horizontal and oblique fissures underwent partial excision of the pericyst and obliteration of the residual cavity by vertically placed imbricating sutures between the walls of adjacent lobes, to avoid possible compromise of the lung parenchyma. One patient had capitonnage by circumferential horizontal mattres sutures for residual cavity left in horizontal fissure. The upper lobe puckered, collapsed, and was affected by diffuse hemorrhages, which improved quickly when the sutures were removed and the cavity was left open. Capitonnage by the classic method was therefore abandoned in such a situation, and vertical imbricating sutures were used between the adjacent walls of the contiguous lobes. This
TABLE 1. Clinical features

\begin{tabular}{llr}
\hline & No. & $\%^{*}$ \\
\hline Symptoms & & \\
$\quad$ Chest pain & 9 & 75.0 \\
Cough & 7 & 58.3 \\
Hemoptysis & 6 & 50.0 \\
$\quad$ Expectoration of grape skin & 2 & 16.6 \\
Signs & & \\
$\quad$ Normal chest examination & 7 & 58.3 \\
Features of mass lesion & 4 & 33.3 \\
Incidental radiographic findings & 1 & 8.3 \\
\hline
\end{tabular}

*Percentages do not add to $100 \%$ because more than one clinical feature was frequently seen in a single patient.

method had also the advantage of maintaining the orientation of the relation between two adjacent lobes. Pericystectomy was timeconsuming and associated with significant hemorrhage. Lobectomy was not required because patients with very large or infected cysts, which could require lobectomy, were excluded from the study. The diagnosis of hydatid cyst was confirmed on histopathologic examination of the specimen in all cases.

\section{Discussion}

Hydatid disease is prevalent and widespread in most sheep-rearing countries in Asia, Australia, South America, and Southern Europe. In Kashmir (India), hydatid disease of the lung is one of the most common surgical entities that a thoracic surgeon comes across and carries significant morbidity and mortality, especially in infected cases. The disease is attributed to occupational exposure during farming practices (sheep rearing), particularly in rural areas; ingestion of contaminated vegetables, drinking of egg-contaminated water (containing hexacanth larvae), and traditional intake of mutton and beef. Because the practice of close contact with domestic animals such as dogs is rare in Kashmir, which is Muslim dominated, the sheep-dog association apparently seems to be most commonly implicated in the life cycle of the parasite. Buffalo-dog, goat-dog, cow-dog, and horse-dog associations are also possible.

The liver is commonly affected (75\%), and pulmonary hydatidosis accounts for $15 \%$ of cases. ${ }^{3}$ However, bilateral lung cysts are rare. Salih and associates ${ }^{4}$ reported an analysis of 405 patients: 367 had isolated lung cysts, and 38 had both hepatic and lung cysts. Only 6 of the patients with lung cysts had bilateral disease.

Our objective was to evolve a minimally invasive technique in a single stage for tackling bilateral hydatid lung disease. In our review of the literature, such a surgical approach had generally not been reported for single-stage excision of bilateral hydatid cysts. Bilateral lung hydatid disease has conventionally been managed with two-stage posterolateral thoracotomy. However, Salih and associates ${ }^{4}$ treated bilateral lung hydatid cysts by median sternotomy. This approach was also used for resection of bilateral metastasis by Regal and colleagues. ${ }^{5}$ However, the left lower lobe was difficult to approach through this procedure. Salih and associates ${ }^{4}$ managed bilateral lung cysts preferably by median sternotomy and managed combined pulmonary and hepatic cysts by a transthoracic-transdiaphragmatic approach. Median sternotomy, although a single-stage procedure, was associated with significant morbidity. During our routine practice, we have also found that the 
median sternotomy, although safe for patients with poor lung function, is associated with morbidity from excessive hemorrhage, wound sepsis, sternal osteomyelitis, suture granulomas, persistent sinus, and postoperative pain, all of which disturb the patient's lifestyle. Such an approach cannot be justified in the treatment of a patient with a benign disease such as hydatidosis.

Although our experience is limited, we found many advantages with this technique. This procedure has the benefit of convenience during anesthesia, particularly in high-risk patients with reduced lung reserves. Anesthesia is better tolerated in the supine posture, unlike the lateral approach, and there is less chance of impairment of lung function and consequent hypoxia. In addition, this approach is cosmetically appealing, particularly for young girls. It carries less morbidity and also curtails the hospital stay and expenses. It avoids the social embarrassment of undergoing two consecutive procedures and is economical. In this approach, thoracic muscles such as the latissimus dorsi, pectoralis major, and serratus anterior are preserved. The approach provides easy access to the difficult sites, such as the lower lobes and subpulmonic regions. The upper lobes and central regions can also be approached comfortably, with excellent exposure. Anteriorly, the most appropriate intercostal space can be selected and opened after careful study of the radiograph. This approach can also be converted to lateral thoracotomy in case of emergency. Even though it is bilateral, this procedure causes negligible bleeding relative to the conventional approach, unless the surgeon opts for pericystectomy. In contrast to the median sternotomy and lateral approach, the chest trauma is limited to the fifth intercostal space, instead of involving multiple dermatomes. The severity of the postoperative pain usually associated with the conventional approach was much less in our patients. We did not notice any significant postoperative atelectasis, which is attributed to intraoperative lung manipulation, reflex inhibition of diaphragmatic activity, chest pain, and muscle fatigability resulting in diminished inspiratory efforts, in contrast to posterolateral thoracotomy. In the latter case, regardless of the mechanism, the atelectasis and other complications are undoubtedly enhanced. The vital capacity and peak flow measurements are reduced, and the physiology of ventilation-perfusion is significantly altered because the dependent lung provides most of the gas exchange. We did not notice any significant increase in the length of the procedure.

\section{Conclusion}

We believe that single-stage bilateral minithoracotomy for bilateral hydatid lung disease is a workable treatment option that is minimally invasive by all standards. It is convenient for the surgeon, the anesthesiologist, and the patient. After it has been given further trial, its indications can be expanded to other benign diseases and selected cases of malignant disease. However, its role in the future remains to be determined by a large-scale prospective evaluation.

We thank Gulzar Ahmad Gujri, lrshad Ahmad Shalla, and Ghulam Ahmad Dar for their documentation and illustration.

\section{References}

1. Balikian JP, Mudarris FF. Hydatid disease of the lungs: a roentgenologic study of 50 cases. Am J Roentgenol Radium Ther Nucl Med. 1974;122:692-707.

2. Morris DL, Richards KS. Text book of hydatid disease' current medical and surgical management. 1st ed. London: Butterworth-Heinemann; 1992. p. 25-30.

3. Gossios KJ, Kontoyiannis DS, Dascalogianaki M, Gourtsoyiannis NC. Uncommon locations of hydatid disease. Eur J Radiol. 1997;7:1303-8.

4. Salih OK, Topcuoglu MS, Celik SK, Ulus T, Tokcan A. Surgical treatment of hydatid cysts of the lung: analysis of 405 patients. Can J Surg. 1998;41:131-5.

5. Regal A, Reese P, Antikowiak J, Hart T, Takita H. Median sternotomy for metastatic lung lesions in 131 patients. Cancer. 1985;55:1334-9. 